\title{
Current perspectives on the dynamics of antibiotic resistance in different reservoirs
}

\author{
Manuela Caniça $^{\mathrm{a}, *}$, Vera Manageiro a,b ${ }^{\mathrm{a}}$, Daniela Jones-Dias ${ }^{\mathrm{a}, \mathrm{b}}$, Lurdes Clemente ${ }^{\mathrm{c}}$, \\ Eduarda Gomes-Neves ${ }^{b, d}$, Patrícia Poeta ${ }^{e}$, Elsa Dias ${ }^{a, b, f}$, Eugénia Ferreira ${ }^{a}$ \\ ${ }^{a}$ National Reference Laboratory of Antibiotic Resistances and Healthcare Associated Infections, National Institute of Health Dr. Ricardo Jorge, Lisbon, Portugal \\ ${ }^{\mathrm{b}}$ Centre for the Studies of Animal Science, Institute of Agrarian and Agri-Food Sciences and Technologies, Oporto University, Oporto, Portugal \\ ${ }^{\mathrm{c}}$ INIAV - Instituto Nacional de Investigação Agrária e Veterinária, Lisbon, Portugal \\ ${ }^{\mathrm{d}}$ ICBAS, Instituto de Ciências Biomédicas Abel Salazar, Universidade do Porto, Oporto, Portugal \\ ${ }^{\mathrm{e}}$ Department of Animal and Veterinary Sciences (CECAV), University of Trás-os-Montes and Alto Douro (UTAD), Vila Real, Portugal \\ ${ }^{\mathrm{f}}$ Department of Environmental Health, National Institute of Health, Lisbon, Portugal
}

Received 2 December 2014; accepted 23 July 2015

Available online 4 August 2015

\begin{abstract}
Antibiotic resistance consists of a dynamic web. In this review, we describe the path by which different antibiotic residues and antibiotic resistance genes disseminate among relevant reservoirs (human, animal, and environmental settings), evaluating how these events contribute to the current scenario of antibiotic resistance. The relationship between the spread of resistance and the contribution of different genetic elements and events is revisited, exploring examples of the processes by which successful mobile resistance genes spread across different niches. The importance of classic and next generation molecular approaches, as well as action plans and policies which might aid in the fight against antibiotic resistance, are also reviewed.
\end{abstract}

(C) 2015 Institut Pasteur. Published by Elsevier Masson SAS. All rights reserved.

Keywords: Antibiotics; Resistome; Mobilome; Reservoirs; Dissemination

\section{Introduction}

Many classes of antibiotics are not only clinically valuable in human medicine, but also in other fields such as veterinary medicine and animal food production, including aquaculture $[1,2]$. The agricultural setting also plays an important role in the spread of antibiotic residues in the environment due to

\footnotetext{
* Corresponding author. National Reference Laboratory of Antibiotic Resistances and Healthcare Associated Infections, Department of Infectious Diseases, National Institute of Health Dr. Ricardo Jorge, Av. Padre Cruz, 1649016 Lisbon, Portugal. Tel./fax: +351 217519246.

E-mail addresses: manuela.canica@insa.min-saude.pt (M. Caniça), vera. manageiro@insa.min-saude.pt (V. Manageiro), daniela.dias@insa.min-saude. pt (D. Jones-Dias), lurdes.clemente@iniav.pt (L. Clemente), emneves@ icbas.up.pt (E. Gomes-Neves), ppoeta@utad.pt (P. Poeta), elsa.dias@insa. min-saude.pt (E. Dias), eugenia.ferreira@insa.min-saude.pt (E. Ferreira).
}

their use as additives and biocides in crops. Consequently, all adjacent natural environments consisting of water, soil and plants are environmental niches to be taken into consideration in the dynamics of antimicrobial resistance [3,4].

The use of antibiotics may have dangerous long-term effects that extend beyond selection of specific resistance mechanisms [5]. The selection pressure applied to bacterial communities via widespread discharge of antibiotic residues in the environment strongly contributes to the exposure of several niches to antibiotic-resistant bacteria (commensal and/or pathogenic) [6]. For example, use of animal manure may enhance viable antibiotic-resistant coliform bacteria in soil, increasing the frequency of detection of some antibiotic resistance genes [3]. These bacteria may reach the food chain, since vegetables are grown in soil [7]. Acquired antibiotic resistance is also frequent among isolates from wild animals 
which represents a niche of concern. Indeed, many reports point to wild animals as reservoirs of resistant determinants that commonly appear in other habitats, namely in human settings [8].

It should also be noted that bacterial resistance to antibiotics is related to soil and aquatic native microorganisms which may be producers of antimicrobial compounds $[9,10]$. Several other factors contribute to antibiotic resistance. Indeed, the existence of major anthropogenic actions such as international travel and global trade in foodstuffs strongly contribute to its amplification [11-14] (Table 1). A classical example of a vehicle for transmission of antibiotic-resistant bacteria is the human hand, which can become easily contaminated by environmental surfaces near patients in hospitals or animals in husbandry settings [15,16]. Consequently, the World Health Organization is strongly committed to making people aware of the problem of antibiotic resistance, and especially caretakers, namely through promoting hand hygiene for fighting antibiotic resistance [16,17].

Table 1

Antibiotic resistance is generated by several factors (adapted from [11-13]).

Factors depending on biological and physical influences:

Human activities

Animals (namely insects, birds, wildlife)

Water

Environmental changes

Wind

Changes in geographic localization of bacteria

Factors dependent on humans and their management of antibiotics:

Preservation of ecosystems (eventually bioremediation)

Intensive farming

Sanitation and hygiene measures

Runoff and leak

Manure

Demographic changes (increasing number of elderly people)

Anthropogenic contacts

Socioeconomic factors

Bioterrorism (biological war)

Travel of people and foodstuffs

Patient movement within and between medical institutions

Infection control measures (prevention of infection)

Appropriateness of use

Factors related to the antibiotic itself

Antibiotic use

Novel antibiotics

Dose of treatment

Duration of treatment

Antibiotic residues

Food additives

Selection of antibiotic resistant bacteria

Factors related to microorganisms

Wide spread of commensal bacteria

Extensive spread of old or new pathogens

Higher number of infections (opportunistic)

Increased number of host-pathogen contacts

Modification of microbial diversity

Factors related to the genetic basis of resistance

Cross selection

Non-antibiotic selection

Gene transfer

Clonal spread
There exists an urgent need to elucidate possible connections between antibiotics, environmental organisms and associated bacterial communities, as they may threaten diverse ecosystems and consequently human health [18]. In this review, we emphasize that these settings are linked and may constitute reservoirs of antibiotic resistance determinants, playing important roles in their dynamics.

\section{Antibiotic residues versus resistomes in the environment}

Before the antibiotic era, environmental antibiotic-resistant bacteria already existed, carrying genes that became critically important in medicine [19]. Indeed, for many years the environment consisted of an under-recognized reservoir of resistance genes that have the potential to be transferred and emerge in clinically important bacteria [20-22]. Groh et al. (2006) showed that homologues of multidrug resistance genes present in bacterial pathogens are essential for sediment fitness in non-pathogenic bacteria, by conferring an ecological advantage on these microorganisms [23].

Several reports have demonstrated the existence of antibacterial activity in extracts from different microorganism genera/species against distinct bacteria. A recent study showed that some antibiotic-resistant Gram-negative strains recovered from an industrial alpine location highly polluted with oil hydrocarbons had the ability to produce antimicrobial compounds active against Actinobacteria and Gammaproteobacteria. Thus, the selection pressure present in this environment could lead not only to high antibiotic resistance, but also to the capacity of this population to produce antimicrobial compounds [24]. LeBel et al. (2013) also demonstrated that the heat-stable bacteriocin nisin (naturally produced by Lactococcus lactis) displayed antimicrobial activity against the emerging zoonotic agent Streptococcus suis [25].

In fact, several studies have demonstrated that freshwater/ marine bacteria are also able to produce antibacterial compounds that exhibit antimicrobial activity similar to standard drugs, which is the case for cyanobacteria [26]. In addition, it was demonstrated that extracts from Anabaena spp. were effective against vancomycin-resistant Staphylococcus aureus [27]. Some authors consider that cyanobacteria antibacterial activity is more effective against Gram-positive bacteria $[28,29]$ than Gram-negative, attributed to the protection conferred by the lipopolysaccharide barrier of the Gramnegative cell wall [28]; however, some Gram-negative bacteria, including pathogenic species, are also affected by cyanobacterial compounds. The potential application of bacterial compounds to the development of new antimicrobials therefore appears to be a promising research area.

The relationship between bacteria and antibiotics may be approached in a variety of ways. In the case of cyanobacteria, considering their ubiquity and importance in the ecosystems [30], increasing concern has been attributed to the effects of environmental stressors in these bacteria. In fact, although cyanobacteria can easily adapt to different environmental conditions, they can also be severely affected by 
environmental changes and water contaminants [31,32]. On the other hand, and given that cyanobacteria are exposed to antibiotics and resistant bacteria in their natural habitats [18], we hypothesize that they are able to develop antibiotic resistance mechanisms. Their ability to produce a variety of bioactive antibacterial compounds with potential pharmaceutical interest [26] suggests that they may have developed defense mechanisms against their own toxicity [33].

The role of antibacterial-compound-producing bacteria is a challenge to the scientific community, since these new molecules may constitute a promising future source of antimicrobials. Thus, further research will be needed to understand the effect of such genera/species on the resistome.

\section{The mobilome associated with antibiotic resistance genes}

Genomic events constitute a central process in the mobilization of genetic elements and associated mobile antibioticresistance-encoding genes in different settings [34]. The movement of bacteria from the environment to animals and humans (and vice-versa) contributes to an increase in the mobilome (mobile gene pool) [35]. These genetic exchanges have been significantly reported among human and animal guts [36]. Indeed, lateral gene transfer and recombination of genetic material within bacterial populations strongly contributes to the diversity and adaptability of strains to different environments (Fig. 1).

The wide divergence within specific functional genes, the creation of mosaic-structured genomic regions, as well as the high prevalence of mobile genetic elements contribute to the success of different gene pools, producing new worldwide dispersed hotspots $[5,14,20,34]$. For instance, the acquisition of a single plasmidic but pleiotropic gene that encodes resistance to two structurally and functionally different classes of

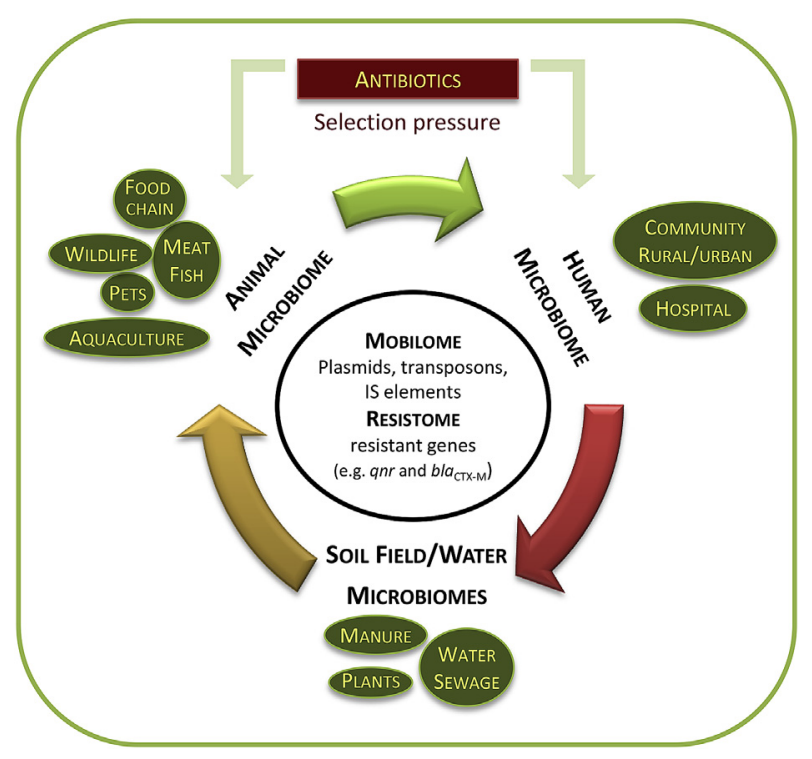

Fig. 1. Crosswalk between resistome and mobilome in different environments. antibiotics also highlights the remarkable adaptive nature of Gram-negative bacteria [37].

In general, mobile genetic elements such as plasmids, insertion sequences (ISs), transposons, genomic islands and phages, constitute the arsenal of bacterial genomes in terms of genetic transfer, contributing to the emergence of novel genotypic and phenotypic variants [35,38,39]. Frequently, these genetic structures may be organized in cascade-like arrangements, contributing to the amplification of mobilization events.

Several reports revealed that mutations in the promoter region and acquisition of functional promoters can turn on a silent gene [40]. For example, IS may disrupt open reading frames and activate gene expression through transposition. A functional promoter may be created through the -35 promoter-like sequence existing in the terminal of some IS elements [40].

The mobilome and the resistome usually follow parallel paths. The environment is broadening the origin of antibiotic resistance genes. This was the case for Kluyvera spp., commensal bacteria of both humans and animals, which went ahead to the mobilization of their chromosomal CTX-M-type $\beta$-lactamase-encoding genes into the plasmids of other bacteria. Thus, CTX-M extended-spectrum $\beta$-lactamase (ESBL) has its origin in Kluyvera spp., which possibly shares other genes with enteric bacteria [41]. The understanding that genes from non-related species might be expressed in new hosts is now evident $[6,8,9]$. Another example is OXA-type- $\beta$-lactamases. Interestingly, the genes encoding these enzymes have already been described in plasmids prior to the human use of antibiotics, since they have moved through horizontal gene transfer between bacterial phyla for millions of years [42]. Recently, an OXA carbapenemase (from Ambler class D) that supposedly had its origin in Turkey (OXA-48) has been described in Europe and in the USA among Klebsiella pneumoniae isolates, causing considerable morbidity and mortality [43,44]. The first report of Shewanella xiamenensis carrying a $b l a_{\text {OXA-48-like }}$ gene suggested that the emergence of different genes from this group had their origin in different S. xiamenensis strains [45]. The contribution of different mobile genetic elements and mechanisms to the dissemination of these carbapenemase-encoding genes $\left(b l a_{\text {OXA-48-like }}\right)$, from Shewanella spp. to Enterobacteriaceae and to other Gram-negative bacteria, has been reported [46]. These antibiotic resistance genes constitute one of the greatest threats in terms of public health because of their ability to resist carbapenems $[43,47]$.

The idea that some resistance mechanisms, such as carbapenemase production, are only linked to infections and human healthcare facilities is no longer valid [48]. The efficacy of $b l a_{\text {OXA-48-like }}$ gene transfer between bacterial species in human cases [44] has also been reported in food-producing, companion and wild animals, as well as in natural environments $[45,49,50]$, highlighting its importance in the dissemination of antibiotic resistance among different reservoirs.

International travel and medical tourism have rapidly driven the resistance mechanisms into an alarming public health problem [51,52]. Apart from the enzymes belonging to Ambler class $\mathrm{D}$, this is also happening with other carbapenemases, such 
as KPC ( $\beta$-lactamases from Ambler class A) and NDM (metalo- $\beta$-lactamase from Ambler class B) [51].

\section{Investigating antibiotic resistance}

Routine investigation of the most relevant antibiotic resistance mechanisms (such as ESBLs, carbapenemases, methicillin-resistant $S$. aureus/MRSA and vancomycinresistant enterococci/VRE) is becoming common in the human sector; however, it is still scarce in the area of food, animals and the environment. The specific detection of ESBLand carbapenemase-producing bacteria in the environment, on farms, in food and companion animals urgently requires means to contain these resistance mechanisms. This is a matter of concern, as carbapenems are currently one of the last resources for treat complicated human infections caused by multidrug resistant Gram-negative bacteria [48].

The colonization of healthy animals may represent a silent source of these resistance mechanisms and a possible transmission pathway to humans via the food chain. On the other hand, the carriage of such resistance mechanisms by healthy humans may also cause their transmission to non-human sources. Moreover, infection and colonization are higher among animal owners, farmers and veterinarians. They can be carriers and transmit bacterial antibiotic resistance genes to animals, namely due to prior selection pressure caused by hospitalization, antibiotic consumption, contact with healthcare settings and/or international voyages (Fig. 1) [49].

Data collection and analysis of antibiotic resistance will improve detection of outbreaks (at a micro-level) and support decision-making (at a macro-level), highlighting the importance of the One-Health approach to combat its global rise in human infectious diseases [17,53].

Our capacity to contain the increase and dissemination of resistance mechanisms such as carbapenemase-producing bacteria will eventually indicate the efficiency of antibiotic use and antibiotic resistance containment policies [17,49]. In 2006, the European Union prohibited antibiotics as growth promoters in animal feed, which was an important step in the use of antibiotics for non-medical purposes. However, the antibiotic resistance of foodborne pathogens remains a matter of concern. The notification rates of zoonoses in confirmed human cases in the EU for the year of 2013 showed that Campylobacter, Salmonella and Escherichia coli were the most frequent bacteria causing human foodborne zoonoses. Despite all efforts, these pathogens still present high levels of resistance to antibiotics used in humans [54]. About 30\% of human Salmonella spp. isolates exhibited multidrug resistance [54].

Emerging antibiotic-resistant foodborne pathogens of animal origin are forcing food and veterinary microbiology laboratories to be increasingly alert due to the impact of these bacteria can on public health. Next-generation sequencing technologies have greatly accelerated the rate and reduced the cost of genomic data acquisition. Whole-genome sequencing (WGS) and molecular epidemiology studies constitute important assets for exploring the bacterial genomes
$[22,39,55]$. The possibility of performing comparative genomic analysis in short periods of time can enable rapid detection of resistance genes that, in turn, may be helpful for distinguishing different bacterial subpopulations $[22,55,56]$.

These methodologies are very useful for monitoring the mobilome, as mobile antibiotic resistance genes are frequently clustered in complex genetic arrays. For instance, hitchhiking genes, such as antibiotic resistance and heavy metal or disinfectant resistance genes, are commonly genetically associated. Moreover, there exist genomic regions that enable the gathering of antibiotic resistance genes, such as integrons and those responsible for their transfer, like plasmids and conjugative transposons [40]. Plasmidome sequencing is also an interesting approach for evaluating antibiotic resistance genes that may spread among different settings [35]. Likewise, a complete proteome might be obtained through matrix-assisted laser desorption/ionization mass spectrometry (MALDI-TOF MS). Correia et al. (2014) reported confirmation of several proteins in $S$. Typhimurium phage type 104 clinical strain through this method, emphasizing the presence of the $\operatorname{Aac}\left(6^{\prime}\right)$ Ib-cr enzyme responsible for both plasmid-mediated aminoglycoside and quinolone resistance [37,57].

Likewise, high-throughput sequencing of nine genomes was used for rapid identification of data in an outbreak caused by enteroaggregative verocytotoxin-producing $E$. coli O104:H4 (STEC) associated with consumption of raw vegetables $[55,58]$. This outbreak was responsible for a hemolytic uremic syndrome in patients, due to TEM- and CTX-M-15producing STEC isolates. The exposure path involved in this outbreak may have been more than one [55,58]: initially, a primary human infection might have arisen from consumption of contaminated food or direct contact with an animal carrying STEC strains; next, subsequent infection might have occurred by the fecal-oral pathway, through manipulation of contaminated vegetables $[55,58]$.

Using the WGS approach, two independent human farm cases of $m e c C$-MRSA infection directly linked to a livestock (cows and sheep) reservoir, were identified in Denmark, supporting zoonotic spread. In these cases, it was demonstrated that the CC130 MRSA lineage was transmitted between animals and humans and that livestock may be a reservoir for MRSA [56].

Thus, new-generation approaches enable a better understanding of specific resistance mechanisms, permitting effective control of complex epidemiological situations. Overall, the use of many types of "omic" approaches is already providing more advanced hypotheses, mechanisms and models of antibiotic resistance evolution [59].

\section{Strengthening the combat against antibiotic resistance}

Actions should be taken to diminish the selection pressure imposed by antibiotics in human communities. Other priority actions should include risk management to minimize antibiotics and antibiotic-resistant bacteria in animals (companion and food-producing) and in the environment, namely in fresh and wastewater. 
Several measures are necessary to fight antibiotic-resistant bacteria, but the main concern involves putting them into practice in all countries, regions and settings [17,53,54,60,61].

Council Recommendation/2002/77/EC on the prudent use of antimicrobial agents in human medicine highlights the need for a "relationship between the occurrence of antimicrobial resistance in certain human pathogens and their occurrence in animals and the environment" [60]. In addition, we must be aware of the fact that "coordination between human, veterinary and environment sectors should be ensured and the magnitude of the relationship between the occurrence of antimicrobial resistant pathogens in humans, animals and the environment should be further clarified and therefore this recommendation does not preclude further initiatives in other areas." [60]. Recommendations from other entities reinforce the need to fight against the rising threats of antibiotic resistance, through publication of detailed action plans and ideas underlining important pressure points $[17,62]$. We also point out that the Transatlantic Task Force on Antimicrobial Resistance of 2014 issued recommendations for future collaboration between the USA and the EU. In this document, strategies for improving the pipeline of new antibacterial drugs was emphasized [63]. The absence of new antibiotics is the major drawback in the field of antibiotic resistance [64].

The implementation of DC $613 / 2013$ by EU countries concerning antimicrobial resistance monitoring in zoonotic and commensal agents in 2014 in food-producing animals and meat for a period of seven years (2014-2020), using standardized validated antimicrobial susceptibility testing methods and harmonized interpretive criteria, will complement and provide early warning of changes in resistance patterns in animal populations, and will monitor future trends in the occurrence of antimicrobial resistance [65].

Many European programs have also been developing policies for diminishing antibiotic use and antibiotic resistance genes in different sources, namely water [66]. In water, reservoirs are highlighted by the recent COST action that takes into account the problem of antibiotics and their consequences, the main objective of which is to make possible wastewater reuse [http://www.nereus-cost.eu/].

Meanwhile, national strategies for combatting antibioticresistant bacteria, identifying priorities and coordinating investments were recently reviewed in the USA [67]. The evaluation of antibacterial products used as feed additives and the impact of antimicrobial resistance on antibiotics of human and veterinary importance have also been revised [68].

\section{Conclusions}

Antibiotic resistance constitutes a health crisis that has consequences throughout the world, striking several settings simultaneously: humans, animals and natural environments. Until the decade of the 90 's, the majority of drugs, including antibiotics, were derived from natural products; since then, a substantial increase in the use of synthetic and semi-synthetic substances as therapeutic agents has occurred [69]. However, the major approach of the pharmaceutical industry to overcoming this problem was an improvement in pre-existing antibiotics to the detriment of research on new molecules [69]. In the future, special attention should be paid to the potential of new natural antimicrobial products as effective and less toxic alternatives [70].

Overall, actions must be taken to diminish selection pressures imposed by antibiotics, in order to reduce exposure of humans to high rates of antibiotic-resistant strains. Priority actions include risk management to access the use of antibiotics and the presence of resistant bacteria in the different environments. In the end, a higher economic and human investment in the field is necessary.

\section{Conflict of interest}

No conflicts of interest to declare.

\section{Acknowledgments}

The authors thank Fundação para a Ciência e a Tecnologia (Portugal) for research funding grants SFRH/BPD/77981/ 2011, SFRH/BD/80001/2011 and SFRH/BPD/77486/2011 attributed to Elsa Dias, Daniela Jones-Dias and Vera Manageiro, respectively, as well as for funding the project PEstOE/AGR/UI0211/2011-2014 (through the Center for the Studies of Animal Science, Institute of Agrarian and AgriFood Sciences and Technologies, Oporto University, Oporto, Portugal). This work was presented, in part, as an opening lecture plus a keynote at the TEMPH2014-Trends in Environmental Microbiology for Public Health, Lisbon, Portugal.

\section{References}

[1] Marshall BM, Levy SB. Food animals and antimicrobials: impacts on human health. Clin Microbiol Rev 2011;24:718-33.

[2] Garcia-Alvarez L, Dawson S, Cookson B, Hawkey P. Working across the veterinary and human health sectors. J Antimicrob Chemother 2012;67:i37-49.

[3] Heuer H, Schmitt H, Smalla K. Antibiotic resistance gene spread due to manure application on agricultural fields. Curr Opin Microbiol 2011;14:236-43.

[4] Baquero F, Martínez JL, Cantón R. Antibiotics and antibiotic resistance in water environments. Curr Opin Biotechnol 2008;19:260-5.

[5] Gillings MR. Evolutionary consequences of antibiotic use for the resistome, mobilome and microbial pangenome. Front Microbiol 2013;4:4.

[6] Perron GG, Quessy S, Bell G. A reservoir of drug-resistant pathogenic bacteria in asymptomatic hosts. PLoS One 2008;3:e3749.

[7] Marti R, Scott A, Tien YC, Murray R, Sabourin L, Zhang Y, et al. Impact of manure fertilization on the abundance of antibiotic-resistant bacteria and frequency of detection of antibiotic resistance genes in soil and on vegetables at harvest. Appl Environ Microbiol 2013;79:5701-9.

[8] Sousa M, Gonçalves A, Silva N, Serra R, Alcaide E, Zorrilla I, et al. Acquired antibiotic resistance among wild animals: the case of Iberian Lynx (Lynx pardinus). Vet Q 2014;34:105-12.

[9] Zhang X, Li Y, Liu B, Wang J, Feng C, Gao M, et al. Prevalence of veterinary antibiotics and antibiotic-resistant Escherichia coli in the surface water of a livestock production region in Northern China. PLoS One 2014;9:e111026.

[10] DeLorenzo ME, Brooker J, Chung KW, Kelly M, Martinez J, Moore JG, et al. Exposure of the grass shrimp, Palaemonetes pugio, to antimicrobial 
compounds affects associated Vibrio bacterial density and development of antibiotic resistance. Environ Toxicol 2014. http://dx.doi.org/10.1002/ tox.22060.

[11] Barbosa TM, Levy SB. The impact of antibiotic use on resistance development and persistence. Drug Resist Updat 2000;3:303-11.

[12] Martinez JL, Baquero F. Interactions among strategies associated with bacterial infection: pathogenicity, epidemicity, and antibiotic resistance. Clin Microbiol Rev 2002;15:647-79.

[13] Allen HK, Donato J, Wang HH, Cloud-Hansen KA, Davies J, Handelsman J. Call of the wild: antibiotic resistance genes in natural environments. Nat Rev Microbiol 2010;8:251-9.

[14] Gaze WH, Zhang L, Abdouslam NA, Hawkey PM, Calvo-Bado L, Royle J. Impacts of anthropogenic activity on the ecology of class 1 integrons and integron-associated genes in the environment. ISME J 2011;5:1253-61.

[15] Gomes-Neves E, Antunes P, Tavares A, Themudo P, Cardoso MF, Gärtner F, et al. Salmonella cross-contamination in swine abattoirs in Portugal: carcasses, meat and meat handlers. Int J Food Microbiol 2012; 157:82-7.

[16] Park HY, Kim SK, Lim YJ, Kwak SH, Hong MJ, Mun HM, et al. Assessment of the appropriateness of hand surface coverage for health care workers according to World Health Organization hand hygiene guidelines. Am J Infect Control 2014;42:559-61.

[17] WHO. antimicrobial Resistance: Global Report on Surveillance. Geneva: Switzerland; 2014. p. 256.

[18] Martinez JL. Environmental pollution by antibiotics and by antibiotic resistance determinants. Environ Pollut 2009;157:2893-902.

[19] D'Costa VM, King CE, Kalan L, Morar M, Sung WW, Schwarz C, et al. Antibiotic resistance is ancient. Nature 2011;477:457-61.

[20] Wright GD. The antibiotic resistome: the nexus of chemical and genetic diversity. Nat Rev Microbiol 2007;5:175-86.

[21] D'Costa V, McGrann K, Hughes D, Wright G. Sampling the antibiotic resistome. Science 2006;311:374-7.

[22] Allen H, Moe L, Rodbumrer J, Gaarder A, Handelsman J. Functional metagenomics reveals diverse beta-lactamases in a remote Alaskan soil. ISME J 2009;3:243-51.

[23] Groh JL, Luo Q, Ballard JD, Krumholz LR. Genes that enhance the ecological fitness of Shewanella oneidensis MR-1 in sediments reveal the value of antibiotic resistance. Appl Environ Microbiol 2007;73:492-8.

[24] Hemala L, Zhang D, Margesin R. Cold-active antibacterial and antifungal activities and antibiotic resistance of bacteria isolated from an alpine hydrocarbon-contaminated industrial site. Res Microbiol 2014; 165:447-56.

[25] Lebel G, Piché F, Frenette M, Gottschalk M, Grenier D. Antimicrobial activity of nisin against the swine pathogen Streptococcus suis and its synergistic interaction with antibiotics. Peptides 2013;50:19-23.

[26] Singh RK, Tiwari SP, Rai AK, Mohapatra TM. Cyanobacteria: an emerging source for drug discovery. J Antibiot 2011;64:401-12.

[27] Bhateja P, Mathur T, Pandya M, Fatma T, Rattan A. Activity of blue green microalgae extracts against in vitro generated Staphylococcus aureus with reduced susceptibility to vancomycin. Fitoterapia 2006;77:233-5.

[28] Martins RF, Ramos MF, Herfindal L, Sousa JA, Skaerven K, Vasconcelos VM. Antimicrobial and cytotoxic assessment of marine cyanobacteria - Synechocystis and Synechococcus. Mar Drugs 2008;6:1-11.

[29] Kreitlow S, Mundt S, Lindequist U. Cyanobacteria - a potential source of new biologically active substances. J Biotechnol 1999;70:61-3.

[30] Vasconcelos VM, Pereira E. Cyanobacteria diversity and toxicity in a wastewater treatment plant (Portugal). Water Res 2001;35:1354-7.

[31] López-Rodas V, Maneiro E, Costas E. Adaptation of cyanobacteria and microalgae to extreme environmental changes derived from anthropogenic pollution. Limnetica 2006:403-10.

[32] González-Pleiter M, Gonzalo S, Rodea-Palomares I, Leganés F, Rosal R, Boltes K, et al. Toxicity of five antibiotics and their mixtures towards photosynthetic aquatic organisms: implications for environmental risk assessment. Water Res 2013;47:2050-64.

[33] Waksman SA. Antagonistic relations of microorganisms. Bacteriol Rev 1941:5:231-91.
[34] Burrus V, Waldor MK. Shaping bacterial genomes with integrative and conjugative elements. Res Microbiol 2004;155:376-86.

[35] Brown Kav A, Sasson G, Jami E, Doron-Faigenboim A, Benhar I, Mizrahi I. Insights into the bovine rumen plasmidome. Proc Natl Acad Sci U S A 2012;109:5452-7.

[36] Devirgiliis C, Barile S, Perozzi G. Antibiotic resistance determinants in the interplay between food and gut microbiota. Genes Nutr 2011;6:275-84.

[37] Robicsek A, Strahilevitz J, Jacoby GA, Macielag M, Abbanat D, Park $\mathrm{CH}$, et al. Fluoroquinolone-modifying enzyme: a new adaptation of a common aminoglycoside acetyltransferase. Nat Med 2006;12:83-8.

[38] Gomes-Neves E, Manageiro V, Ferreira E, Correia da Costa JM, Caniça M. First description of food-borne Salmonella enterica resistance regions R1 and R3 associated to IS26 elements. Res Microbiol 2015:166.

[39] Leavis HL, Willems RJ, van Wamel WJ, Schuren FH, Caspers MP, Bonten MJ. Insertion sequence-driven diversification creates a globally dispersed emerging multiresistant subspecies of E. faecium. PLoS Pathog 2007;3:e7.

[40] Salyers A, Shoemaker NB. Reservoirs of antibiotic resistance genes. Anim Biotechnol 2006;17:137-46.

[41] Bonnet R. Growing group of extended-spectrum $\beta$-lactamases: the CTXM enzymes. Antimicrob Agents Chemother 2004;48:1-14.

[42] Barlow M, Hall BG. Phylogenetic analysis shows that the OXA $\beta$-lactamase genes have been on plasmids for millions of years. J Mol Evol 2002;55:314-21.

[43] Poirel L, Potron A, Nordmann P. OXA-48-like carbapenemases: the phantom menace. J Antimicrob Chemother 2012;67:1597-606.

[44] Manageiro V, Ferreira E, Pinto M, Caniça M. First description of OXA48 carbapenemase harbored by Escherichia coli and Enterobacter cloacae in a single patient, in Portugal. Antimicrob Agents Chemother 2014;58:7613-4.

[45] Tacão M, Correia A, Henriques I. Environmental Shewanella xiamenensis strains that carry $b l a_{\mathrm{OXA}-48}$ or bla $a_{\mathrm{OXA}-204}$ genes: additional proof for $b l a_{\text {OXA-48-like }}$ gene origin. Antimicrob Agents Chemother 2013;57:6399-400.

[46] Poirel L, Bonnin RA, Nordmann P. Genetic features of the widespread plasmid coding for the carbapenemase OXA-48. Antimicrob Agents Chemother 2012;56:559-62.

[47] Nordmann P, Dortet L, Poirel L. Carbapenem resistance in Enterobacteriaceae: here is the storm! Trends Mol Med 2012;18:263-72.

[48] Patel G, Bonomo RA. "Stormy waters ahead": global emergence of carbapenemases. Front Microbiol 2013;4:48.

[49] Woodford N, Wareham DW, Guerra B, Teale C. Carbapenemase-producing Enterobacteriaceae and non-Enterobacteriaceae from animals and the environment: an emerging public health risk of our own making? J Antimicrob Chemother 2014;69:287-91.

[50] Stolle I, Prenger-Berninghoff E, Stamm I, Scheufen S, Hassdenteufel E, Guenther S, et al. Emergence of OXA-48 carbapenemase-producing Escherichia coli and Klebsiella pneumoniae in dogs. J Antimicrob Chemother 2013;68:2802-8.

[51] Cantón R, Akóva M, Carmeli Y, Giske CG, Glupczynski Y, Gniadkowski M, et al. Rapid evolution and spread of carbapenemases among Enterobacteriaceae in Europe. Clin Microbiol Infect 2012;18:413-31.

[52] Leonard DA, Bonomo RA, Powers RA. Class D $\beta$-lactamases: a reappraisal after five decades. Acc Chem Res 2013;46:2407-15.

[53] Mills G. One Health: antimicrobial resistance and disease control: making one health work. Vet Rec 2014;175:473-4.

[54] EFSA/ECDC. The European Union summary report on antimicrobial resistance in zoonotic and indicator bacteria from humans, animals and food in 2013. EFSA J 2015;13:4036. 178 pp.

[55] Ho CC, Yuen KY, Lau SK, Woo PC. Rapid identification and validation of specific molecular targets for detection of Escherichia coli O104:H4 outbreak strain by use of high-throughput sequencing data from nine genomes. J Clin Microbiol 2011;49:3714-6.

[56] Harrison EM, Paterson GK, Holden MTG, Larsen J, Stegger M, Larsen AR, et al. Whole genome sequencing identifies zoonotic 
transmission of MRSA isolates with the novel mecA homologue mecC. EMBO Mol Med 2013;5:509-15.

[57] Correia S, Nunes-Miranda JD, Pinto L, Santos HM, de Toro M, Sáenz Y, et al. Complete proteome of a quinolone-resistant Salmonella Typhimurium phage type DT104B clinical strain. Int J Mol Sci 2014;15:14191-219.

[58] EFSA. Urgent advice on the public health risk of Shiga-toxin producing Escherichia coli in fresh vegetables. EFSA J 2011;9:2274.

[59] Franzosa EA, Hsu T, Sirota-Madi A, Shafquat A, Abu-Ali G, Morgan XC, et al. Sequencing and beyond: integrating molecular 'omics' for microbial community profiling. Nat Rev Microbiol 2015;13:360-72.

[60] European Council. Council Recommendation of 15.11.2001 on the Prudent Use of Antimicrobial Agents in Human Medicine (2002/77/EC). OJ L34 of 5.2.2002, p.13. Brussels, Belgium: European Council (EC); 2001.

[61] Review on Antimicrobial Resistance. Antimicrobial Resistance: Tackling a Crisis for the Health and Wealth of Nations. 2014.

[62] Bronzwaer S, Lönnroth A, Haigh R. The European Community strategy against antimicrobial resistance. Euro Surveill 2004;9:30-4.

[63] CDC: Centers for Disease Control and Prevention. Transatlantic Taskforce on Antimicrobial Resistance: Progress Report (TATFAR). 2014.
[64] Livermore DM. Discovery research: the scientific challenge of finding new antibiotics. J Antimicrob Chemother 2011;66:1941-4.

[65] Commission Implementing Decision 613/2013 of 12.11.2013 on the monitoring and reporting of antimicrobial resistance in zoonotic and comensal bacteria.

[66] Fatta-Kassinos D, Manaia C, Berendonk TU, Cytryn E, Bayona J, Chefetz B, et al. COST action ES1403: new and emerging challenges and opportunities in wastewater reuse (NEREUS). Environ Sci Pollut Res Int 2015:22:7183-6.

[67] The Whitehouse. National Strategy for Combating Antibiotic-resistant Bacteria. 2014.

[68] EFSA Panel on Additives and Products or Substances used in Animal Feed (FEEDAP). Guidance on the assessment of bacterial susceptibility to antimicrobials of human and veterinary importance. EFSA J 2012;10:2740.

[69] Li JW, Vederas JC. Drug discovery and natural products: end of an era or an endless frontier? Science 2009;325:161-5.

[70] Singh SB, Barrett JF. Empirical antibacterial drug discovery-foundation in natural products. Biochem Pharmacol 2006;71:1006-15. 